\title{
Injeção epidural preventiva de xilazina ou amitraz em eqüinos: efeitos clínicos e comportamentais
}

\author{
Pre-emptive epidural injection of xylazine or amitraz, in horses: clinical and behavioral effects
}

\author{
Erica Cristina Bueno do Prado Guirro ${ }^{\text {I* }}$ Isabella Maria Marchesini Ferreira ${ }^{\mathrm{II}}$ \\ Guilherme Roberto Sobrinho"II Carlos Augusto Araújo Valadão ${ }^{\mathrm{IV}}$
}

\section{RESUMO}

A xilazina é o fármaco agonista adrenérgico $\alpha_{2}$ mais utilizado pela via epidural de eqüinos e sabe-se que o amitraz tem ação sobre esses receptores. Assim, foram avaliados os efeitos clínicos e comportamentais causados pela injeção epidural de $0,17 \mathrm{mg} \mathrm{kg}^{-1}$ de xilazina $(G X)$ ou de $0,1 \mathrm{mg} \mathrm{kg}^{-1}$ de amitraz diluído em emulsão lipídica (GA) durante 24 horas, em doze eqüinos. A freqüencia cardíaca e a altura de cabeça mantiveram-se inalteradas; a freqüencia respiratória aumentou de T105 a T360 no GX; a temperatura retal elevou-se de T120 a T720 em GA e de T360 a T720 em GX. Não houve diferença no tempo de latência para urinar ou defecar em ambos os grupos. Em relação às alterações comportamentais, o amitraz provocou sedação (50\%), ptose labial (33,4\%) e palpebral $(16,7 \%)$, enquanto que a xilazina promoveu ataxia (50\%), sudorese perineal $(33,4 \%)$, ptose palpebral $(16,6 \%) e$ relaxamento do esfíncter anal (16,6\%). Considera-se segura a injeção epidural de xilazina ou amitraz em eqüinos, pois as alterações clínicas e comportamentais promovidas são leves $e$ não limitam seu uso.

Palavras-chave: amitraz, analgesia, epidural, eqüinos, xilazina.

\section{ABSTRACT}

Xylazine is the $\alpha_{2}$-adrenergic agonist more employed by epidural route in horses and it is known that amitraz acts on these receptors. So, clinical and behavioral effects caused by epidural injection of $0.17 \mathrm{mg} \mathrm{kg}^{-1}$ xylazine $(G X)$ or $0.1 \mathrm{mg} . \mathrm{kg}^{-1}$ amitraz diluted in lipidic emulsion (GA) were evaluated in 12 horses. Heart rate and height of the head didn't change; respiratory rate increased from T105 to T360 in
GX; rectal temperature increased from $T 120$ to T720 in GA and from T360 to T720 in GX. There is no difference in latency to urine or defecate in both groups. Concerning behavioral changes, amitraz promoted sedation (50\%), lip (33.4\%) and eye (16.6\%) dropping, while xylazine caused ataxia (50\%), perineal sweating (33.4\%), eye dropping (16.6\%) and anal sphincter relaxation (16.6\%). So, epidural injection of xylazine or amitraz was considered safe because the clinical and behavioral changes observed are subtle and don't limit its application.

Key words: amitraz, analgesia, epidural, horses, xylazine.

\section{INTRODUÇÃo}

Até recentemente a analgesia de eqüinos foi negligenciada e, ainda hoje, muitos clínicos negam o uso de analgésicos. Entretanto, este posicionamento opõe-se aos novos conceitos humanitários, éticos e de bem-estar animal e é facilmente refutado com base na farmacologia de diferentes protocolos analgésicos (TAYLOR, 2005). Além disso, a presença de dor no período pós-operatório imediato gera efeitos adversos na qualidade de vida do paciente (WU et al., 2003).

A administração epidural de analgésicos em eqüinos permite a execução de intervenções na região urogenital sem a necessidade de submeter o animal à anestesia geral (LE BLANC \& CARON, 1990; SKARDA, 1991; DOHERTY et al., 1997). A xilazina é o

IPrograma de Pós-graduação em Cirurgia Veterinária, Faculdade de Ciências Agrárias e Veterinárias (FCAV), Universidade Estadual Paulista (UNESP), 14884-900, Jaboticabal, SP, Brasil. Campus Palotina, Universidade Federal do Paraná (UFPR), 85950-000, Palotina, PR, Brasil. E-mail: ericaguirro@ufpr.br.*Autor para correspondência.

"IAutônoma, Campinas, SP, Brasil.

IIIMinistério da Agricultura, Pecuária e Abastecimento (MAPA), Iporã, PR, Brasil.

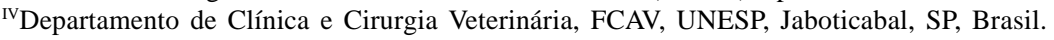


agonista $\alpha_{2}$ mais utilizado em grandes animais e, administrada por via epidural, produz analgesia perineal sem alterar as variáveis cardiorrespiratórias em eqüinos (SKARDA \& MUIR III, 1996a; SKARDA \& MUIR III, 1996b; DE ROSSI \& GÓMEZ DE SEGURA, 2001).

$\mathrm{O}$ amitraz atua nos receptores adrenérgicos $\alpha_{2}$ centrais e periféricos (ROBERTS \& ARGENZIO, 1986; HUGNET et al., 1996; LEE et al., 2003), o que pode ser confirmado pelo sucesso no emprego de antagonistas $\alpha_{2}$ nos quadros de síndrome cólica decorrentes do uso tópico de amitraz (ROBERTS \& SEAWRIGHT, 1979; ROBERTS \& SEAWRIGHT, 1983; AUER et al., 1984). Em eqüinos, o uso sistêmico produz sedação e redução da atividade locomotora e da motilidade intestinal (HARKINS et al., 1997; QUEIROZ NETO et al., 2000) e a injeção epidural gera sedação, miorrelaxamento e mínimas alterações cardiovasculares e respiratórias (VALADÃO, 1998). ALMEIDA et al. (2004) relatam aumento da latência ao estímulo nocivo de origem térmica em bovinos tratados com amitraz pela via epidural.

A maioria dos estudos que verificou os efeitos do amitraz utilizou diluição em dimetilformamida, xileno ou dimetil sulfóxido (SAKATE, 1990; FLÓRIO et al., 1995; VALADÃO, 1998; QUEIROZ NETO et al., 2000; BALESTRERO, 2001; FARIAS et al., 2002; ALMEIDA et al., 2005). Todavia, FARIAS (2004), LINARDI (2004) e MENDES (2004) citam que a diluição em emulsão lipídica garante maior permanência do amitraz no espaço epidural e minimização dos efeitos colaterais decorrentes da absorção sistêmica. Além disso, cães que receberam aplicação epidural de amitraz diluído como uma emulsão lipídica não apresentaram sinais neurológicos e nem lesões histopatológicas na medula (FARIAS, 2004). Assim, este trabalho avaliou os efeitos clínicos e comportamentais promovidos pela administração epidural da xilazina ou do amitraz diluído em emulsão lipídica, em eqüinos.

\section{MATERIAL E MÉTODOS}

O início do experimento foi padronizado às $8 \mathrm{~h}$ a fim de serem evitadas variações decorrentes do ciclo circadiano. Doze eqüinos com peso $318 \pm 48 \mathrm{~kg}$, de três a sete anos, sendo cinco machos castrados e sete fêmeas não-prenhes e fora do período de estro foram avaliados por um observador "cego" quanto à freqüência cardíaca (FC, bpm), freqüência respiratória ( $f$, mpm), temperatura retal $\left(\mathrm{TR},{ }^{\circ} \mathrm{C}\right)$ e altura da cabeça (AC, \% da altura da cabeça até o solo em relação ao tempo basal) em T-30.

Foram infiltrados 3mL de lidocaína 2\% no espaço subcutâneo de $\mathrm{Co}_{1}-\mathrm{Co}_{2}$ e após cinco minutos a pele foi perfurada com agulha hipodérmica previamente à inserção da agulha espinhal de Tuohy até o espaço epidural para permitir a passagem do cateter epidural, que foi introduzido cranialmente por $15 \mathrm{~cm}$ antes da administração dos fármacos conforme o grupo experimental: $0,17 \mathrm{mg} \mathrm{kg}^{-1}$ de xilazina para o grupo GX e $0,1 \mathrm{mg} \mathrm{kg}^{-1}$ de amitraz diluído em emulsão lipídica para o grupo GA, em T0. O volume final das injeções foi ajustado com $\mathrm{NaCl}$ 0,9\%, conforme a equação 3,4mL+ (peso do animal em kg x 0,013mL) (SEGURA et al., 1997) e o tempo de aplicação foi padronizado em dois minutos. Novas mensurações foram realizadas em T15, T30, T45, T60, T75, Т90, Т120, Т240, Т360, Т480, T720, T1080 e T1440. Além disso, foram observados o tempo de latência para urinar e defecar e quaisquer alterações comportamentais verificadas durante o período de 24 horas foram relatadas.

Os valores de FC, f, TR e AC foram submetidos ao teste ANOVA de Uma Via com Repetições Múltiplas entre tempos, seguidos pelo teste de Dunnett $(\mathrm{P} \leq 0,05)$ e, entre grupos, os dados foram submetidos ao teste t $(\mathrm{P} \leq 0,05)$. O tempo de latência para urinar e defecar após as injeções epidurais foi comparado pelo teste $\mathrm{t}(\mathrm{P} \leq 0,05)$.

\section{RESULTADOS E DISCUSSÃO}

Os dados referentes à freqüência cardíaca, freqüência respiratória, temperatura retal e altura de cabeça estão ilustrados na figura 1. Não houve variação significativa nos valores de freqüência cardíaca entre tempos ou entre grupos, possivelmente porque o amitraz diluído em emulsão lipídica e administrado pela via epidural fique restrito ao canal epidural e reduza significativamente a absorção sistêmica do fármaco, visto que após o uso intravenoso geralmente ocorre bradicardia em coelhos (FARIAS et al., 2002), cães (FARIAS et al., 2005) e eqüinos (QUEIROZ NETO et al., 2000; LINARDI, 2004). Após o uso epidural dessa mesma formulação em cães, FARIAS (2004) relatou bradicardia transitória devido, inicialmente, à ativação do reflexo vagal e à liberação de acetilcolina por fibras parassimpáticas e, tardiamente, pela inibição présináptica da liberação de noradrenalina (DOHERTY, 1988; EISENACH et al., 1996). Previamente já foi descrita a manutenção da freqüência cardíaca após a injeção epidural de xilazina em eqüinos (SKARDA \& MUIR III, 1996a; SKARDA \& MUIR III, 1996b; DE ROSSI \& GÓMEZ DE SEGURA, 2001).

Entre tempos, a freqüência respiratória aumentou de T105 a T360 no GX, enquanto em GA não foram observadas mudanças. Não houve variação entre grupos. Em eqüinos, o amitraz aplicado pela via 


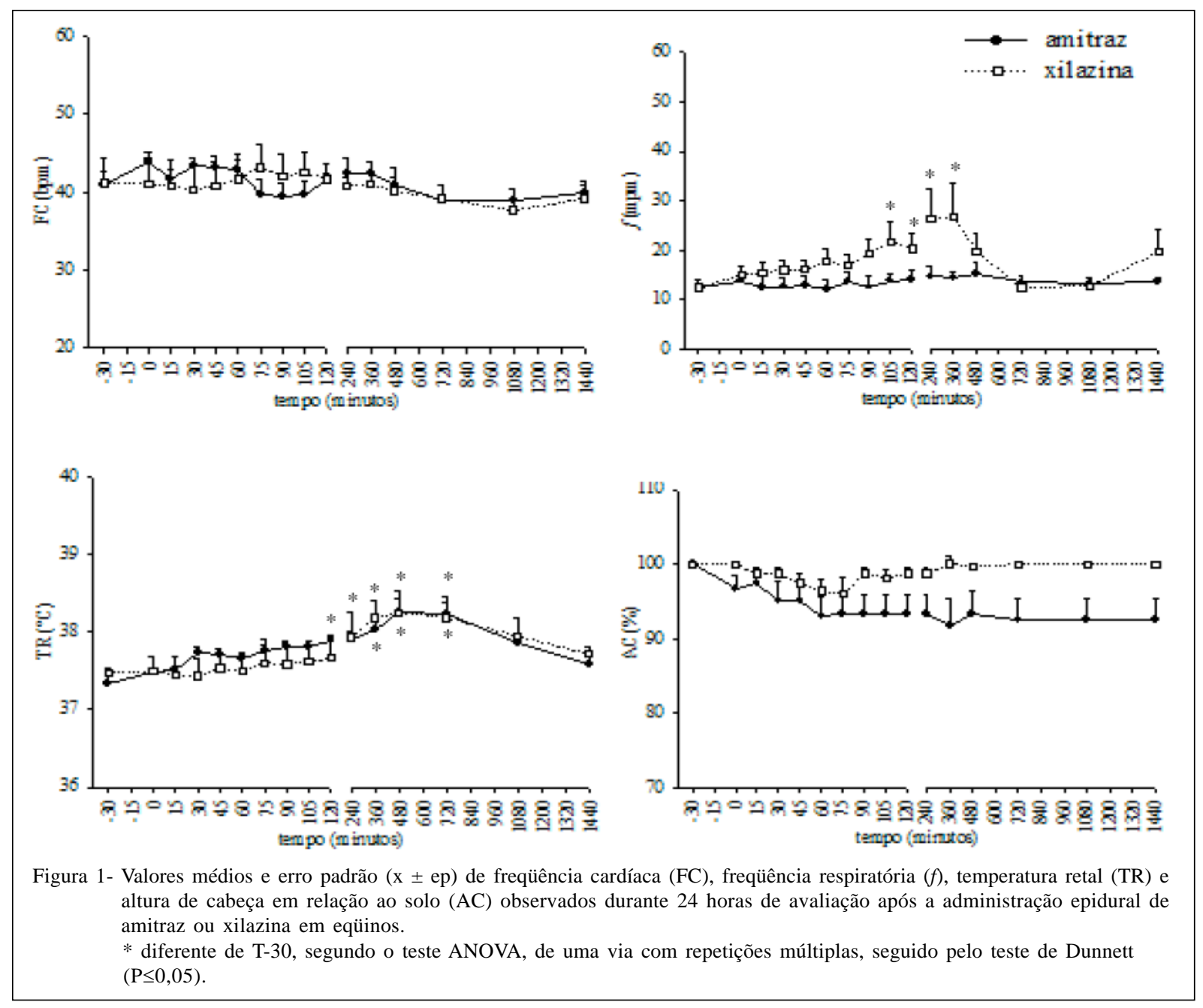

intravenosa pouco interfere nesta variável (QUEIROZ NETO et al., 2000; LINARDI, 2004) e a utilização da via epidural pode minimizar ainda mais essas variações devido à permanência do fármaco no espaço epidural (VALADÃO et al., 1990; DE ROSSI \& GÓMEZ DE SEGURA, 2001). Vale salientar que T105 a T360 ocorreram entre $10 \mathrm{~h} 15 \mathrm{~min}$ e $14 \mathrm{~h} 30 \mathrm{~min}$, respectivamente, e que o presente estudo foi conduzido em condições naturais sem climatização, portanto, é provável que o aumento da freqüência respiratória observado tenha sido influenciado pela temperatura ambiente que superava a média de $29,3^{\circ} \mathrm{C}$.

A temperatura retal, entre tempos, apresentou aumento de T120 a T720 em GA e de T360 a T720 em GX. Não houve variação entre grupos. Os agonistas $\alpha_{2}$ deprimem os mecanismos termorregulatórios e, conseqüentemente, a temperatura corpórea torna-se dependente do meio (LIVINGSTON et al., 1984; VESAL et al., 1998), podendo haver aumento (ST. JEAN et al., 1990; CHEVALIER et al., 2004) ou redução (LIVINGSTON et al., 1984; TIWARI et al., 1998) conforme o ambiente. Assim, é provável que tenha ocorrido ação da temperatura ambiente sobre esta variável e a similaridade das curvas apresentadas pelos dois grupos experimentais sugere tal influência.

A altura de cabeça em relação ao solo não apresentou alteração significativa entre tempos e entre grupos. Este achado deve-se à manutenção do fármaco no interior do canal epidural, pois é relatada a ocorrência de ptose da cabeça após a injeção de amitraz em eqüinos, tratados pela via nasal (BUENO DE CAMARGO et al., 1999) ou intravenosa (QUEIROZ NETO et al., 1998; VALADÃO, 1998; LINARDI, 2004), quando a absorção do fármaco leva à redistribuição em centros superiores e à ativação adrenérgica supraespinhal (EISENACH et al., 1996; OLIVERO VASQUEZ et al., 2000). A manutenção desta variável corrobora LE BLANC \& CARON (1990), que inferem ausência de sedação em eqüinos tratados com xilazina pela via epidural, justificada pela baixa dose do fármaco utilizado 
por esta via, levando a níveis plasmáticos insuficientes para promover a sedação característica do uso sistêmico deste fármaco (LE BLANC \& EBERHART, 1990; SKARDA \& MUIR III, 1996b). Esse parâmetro já foi adotado anteriormente para avaliar o efeito sedativo de agonistas de receptores adrenérgicos do tipo $\alpha_{2}$ (ST. JEAN et al., 1990; SKARDA et al., 1996a; LUNA et al., 1997; POLIMENO et al., 2000; VALADÃO et al., 2003; ALMEIDA et al., 2004).

Em relação às alterações comportamentais dos animais, o amitraz produziu sedação em 50,0\%, ptose labial em 33,4\% e ptose palpebral em 16,7\%, enquanto que a xilazina causou ataxia em 50,0\%, sudorese perineal em 33,4\% e, ainda, ptose palpebral e relaxamento do esfíncter anal em 16,7\% dos animais. Estes dados estão de acordo com estudos anteriores que relatam ter encontrado esses efeitos em eqüinos tratados com agonistas $\alpha_{2}$ pela via epidural devido à absorção sistêmica, mesmo que reduzida (EISENACH et al., 1993; SKARDA \& MUIR III, 1996a; DE ROSSI \& GÓMEZ DE SEGURA, 2001; CHEVALIER et al., 2004), confirmada pela baixa concentração do fármaco no plasma e elevada no fluido cérebro espinhal e na circulação central (CASTRO \& EISENACH, 1989; LE BLANC \& CARON, 1990; GRUBB et al., 2002). O uso sistêmico de agonistas $\alpha_{2}$ proporciona concentrações plasmáticas superiores (LE BLANC \& CARON, 1990).

Não houve diferença significativa no tempo de latência para urinar (GA = 100 $\pm 15 \mathrm{~min}$; GX = $92 \pm 19 \mathrm{~min}$ ), o que difere de alguns relatos (SKARDA \& MUIR III, 1996b; OLIVERO VASQUEZ et al., 2000; LIN et al., 2003), provavelmente devido ao veículo empregado. Também não foi verificada diferença no tempo de latência para defecar (GA = $125 \pm 57 \mathrm{~min}$; GX $=107 \pm 22 \mathrm{~min})$ e ,embora não haja relatos quanto a esse parâmetro, já se verificou que o uso epidural de agonistas $\alpha_{2}$ pode reduzir os quadros de hipomotilidade e de síndrome cólica (LE BLANC et al., 1988; LE BLANC \& EBERHART, 1990; DE ROSSI \& GÓMEZ DE SEGURA, 2001; GOODRICH et al., 2002).

\section{CONCLUSÃO}

Diante do exposto, considera-se segura a administração epidural de xilazina ou do amitraz diluído em emulsão lipídica, em eqüinos, pois as alterações clínicas e comportamentais promovidas são discretas e não limitam sua aplicação.

\section{AGRADECIMENTOS}

À Fundação de Amparo a Pesquisa do Estrado de São Paulo (FAPESP) (processo $\left.n^{\circ} 03 / 0555-5\right)$, pelo apoio financeiro, e à Bayer Saúde Animal, pela doação de Rompum ${ }^{\circledR}$.

\section{COMITÊ DE ÉTICA E BIOSSEGURANÇA}

Este trabalho foi conduzido de acordo com as normas éticas após ser aprovado pelo Comitê de Ética e Bem-estar Animal da FCAV - UNESP (protocolo n-009480).

\section{REFERÊNCIAS}

ALMEIDA, R.M. Avaliação dos efeitos dos amitraz por via epidural em vacas. Braz J Vet Res Anim Sci, v.42, n.6, p.419-428, 2005.

ALMEIDA, R.M. et al. Efeitos da administração epidural de amitraz, xilazina ou dimetil sulfóxido em vacas / Effects of epidural injection of amitraz, xylazine or dimethyl sulfoxide in cows. Arq Bras Med Vet Zootec, v.55, n.6, p.723-732, 2004.

BALESTRERO, L.T. Aspectos farmacológicos da injeção intravenosa ou peridural de amitraz, em eqüinos. 2001. 47f. Dissertação (Mestrado em Cirurgia Veterinária) - Faculdade de Ciências Agrárias e Veterinárias, Universidade Estadual Paulista, Jaboticabal.

BUENO DE CAMARGO, M.H. et al. Sedação de equinos através da aplicação de amitraz por aspersão sobre as mucosas nasais. Ars Veterinária, v.16, n.2, p.177-180, 1998.

CASTRO, M.I.; EISENACH, J.C. Pharmacokinetics and dynamics of intravenous, intrathecal, and epidural clonidine in sheep. Anesthesiology, v. 71, p.418-425, 1989.

CHEVALIER, H.M. et al. Effect of caudal epidural xylazine on intraoperative distress and post-operative pain in Holstein heifers. Vet Anaesth Analg, v.31, p.1-10, 2004.

DE ROSSI, R.; GÓMEZ DE SEGURA, I.A. Efeitos analgésicos, hemodinâmicos e respiratórios da cloridrato de xilazina epidural caudal em eqüinos. A Hora Veterinária, v.120, p.49-53, 2001.

DOHERTY, T.J. Physiologic effects of alpha 2 adrenergic receptors. J Am Vet Med Assoc, v.192, p.1612-1614, 1988.

DOHERTY, T.J. et al. Effect of high volume epidural morphine, ketamine and butorphanol on halothane minimum alveolar concentration in ponies. Equine Vet J., v.29, n.5, p.370-373, 1997.

EISENACH, J.C. et al. á ${ }_{2}$-Adrenergic agonists for regional anesthesia: a clinical review of clonidine (1984-1995). Anesthesiology, v.85, n.3, p.655-674, 1996.

EISENACH, J.C. et al. Hemodynamic and analgesic actions of epidurally administered clonidine. Anesthesiology, v.78, p.277-287, 1993.

FARIAS, A. Avaliação biotelemétrica e farmacológica da administração sistêmica do amitraz, em cães. 2004. 107f. Tese (Doutorado em Cirurgia Veterinária) - Faculdade de Ciências Agrárias e Veterinárias, Universidade Estadual Paulista, Jaboticabal.

FARIAS, A. et al. Monitoramento continuado de coelhos tratados com amitraz ou xilazina por técnica de biotelemetria In: COMBRAVET, 29, 2002, Gramado, RS. Anais... Porto Alegre: Sociedade de Veterinária do Rio Grande do Sul, 2002. Meio Digital - CD. 2002. 
FARIAS, A. et al. Estudo eletrocardiográfico em cães submetidos à aplicação intravenosa de amitraz. Ars Vet, v. 21, supl., p.109-115, 2005.

FLÓRIO, J.C. et al. Effects of amitraz on the arterial blood pressure and body rectal temperature of conscious rats. Braz J Vet Res Anim Sci, v.32, n.3, p.160-164, 1995.

GOODRICH, L.R. et al. Epidural morphine and detomodine decreases postoperative hindlimb lameness in horses after bilateral stifle arthroscopy. Vet Surg, v.31, p.232-239, 2002.

GRUBB, T.L. et al. Caudal epidural analgesia in cattle. Vet Anaesth Analg, v.29, p.64-68, 2002.

HARKINS, J.D. et al. Development and characterization of an equine behaviour chamber and the effects of amitraz and detomidine on spontaneous locomotor activity. J Vet Pharmacol Ther, v.20, p.396-401, 1997.

HUGNET, C. et al. Toxicity and kinetics of amitraz in dogs. Am J Vet Res, v.57, n.10, p.1506-1510, 1996.

LE BLANC, P.H.; EBERHART, S.W. Cardiopulmonary effects of epidurally administered xylazine in the horse. Equine Vet J, v.22, n.6, p.389-391, 1990.

LE BLANC, P.H.; CARON, J.P. Clinical use of epidural xylazine in the horse. Equine Vet J, v.22, n.3, p.180-181, 1990.

LE BLANC, P.H. et al. Epidural injection of xylazine for perineal analgesia in horses. J Am Vet Med Assoc, v.193, n.11, p.1405-1408, 1988.

LEE, I. et al. Antagonistic effects of intravenous or epidural atipamezole on xylazine-induced dorsolumbar epidural analgesia in cattle. Vet J, v.166, p.194-197, 2003.

LIN, H.C. et al. Comparison of the analgesic effect of clonidine and lidocaine when administered epidurally in cattle. Bovine Practitioner, v.37, n.1, p.83-88, 2003.

LINARDI, R.L. Efeitos da xilazina e do amitraz sobre as mensurações ecocardiográficas de eqüinos. 2004. $65 f$ Dissertação (Mestrado em Cirurgia Veterinária) - Faculdade de Ciências Agrárias e Veterinárias, Universidade Estadual Paulista, Jaboticabal.

LIVINGSTON, A. et al. Effects of clonidine and xylazine on body temperature in the rat. Br J Pharmacol, v.81, p.189193, 1984.

LUNA, S.P.L. et al. Efeito da homeopatia sobre a sedação com detomidina em eqüinos. A Hora Veterinária, v.99, p.66-71, 1997.

MENDES, M.C. Avaliação do amitraz e da romifidina em eqüinos. 2004. 53f. Trabalho de Graduação (Conclusão do Curso de Medicina Veterinária) - Faculdade de Ciências Agrárias e Veterinárias, Universidade Estadual Paulista, Jaboticabal.

OLIVERO VÁSQUEZ, Y.I. et al. Utilidad de la clonidina por via peridural em anestesiología. Rev Med Hosp Gen Mex, v.63, n.4, p.261-266, 2000.

POLIMENO, F,C. et al. Estudo dos efeitos do amitraz sobre parâmetros fisiológicos de bovinos. Cienc Anim Bras, v.1, p.210, 2000.

QUEIROZ NETO, A. et al. Characterization of the antinociceptive and sedative effect of amitraz in horses. Vet Pharmacol Therap, v.21, p.400-405, 1998.
QUEIROZ-NETO, A. et al. Effect of amitraz and xylazine on some physiological variables of horses. Arq Bras Med Vet Zootec, v.52, n.1, p.27-32, 2000.

ROBERTS, M.C.; ARGENZIO, A. Effects of amitraz, several opioate derivates and anticholinergic agents on intestinal transit ponies. Equine Vet J, v.18, n.4, p.256-260, 1986.

ROBERTS, M.C.; SEAWRIGHT, A.A. Amitraz induced large intestinal impaction in the horse. Aust Vet J, v.55, p.553554, 1979.

ROBERTS, M.C.; SEAWRIGHT, A.A. Experimental studies of drug-induced impaction colic in the horse. Equine Vet J, v.15, n.3, p.222-228, 1983.

SAKATE, M. Efeitos comportamentais do amitraz (Triatox $^{\circledR}$ ). 1990. 159f. Tese (Doutorado em Farmacologia) - Faculdade de Medicina Veterinária e Zootecnia, Universidade de São Paulo, São Paulo.

SEGURA, I.G. et al. Epidural injection of ketamine for perineal analgesia in the horse. Vet Surg, v.27, n.4, p.384-391, 1997.

SKARDA, R.T. Local anesthetics and local anesthetic techniques in horses. In: MUIR, W.W.; HUBBELL, J.A.E. Equine anesthesia - Monitoring and emergency therapy. St. Louis: Mosby Year Book, 1991. Cap.10, p.199-246.

SKARDA, R.T.; MUIR III, W.W. Analgesic, hemodynamic, and respiratory effects of caudal epidurally administered xylazine hydrochloride solution in mares. Am J Vet Res, v.57, n.2, p.193-200, 1996a.

SKARDA, R.T.; MUIR III, W.W. Comparison of antinociceptive, cardiovascular, and respiratory effects, head ptosis, and position of pelvic limbs in mares after caudal epidural administration of xylazine and detomidine hydrochloride solution. Am J Vet Res, v.57, n.9, p.1338-1345, 1996b.

ST. JEAN, G. et al. Caudal epidural analgesia induced by xylazine administration in cows. Am J Vet Res, v.51, p.1232-1236, 1990 .

TAYLOR, P.M. Pharmacological Approaches to Pain Management in the Horse. In: ANNUAL CONVENTION OF THE AMERICAN ASSOCIATION OF EQUINE PRACTITIONERS, 51., 2005, Seattle. Anais... Seatle: American Association of Equine Practitioners, 2005. Acesso em: 11/01/2008. On line. Disponível em <http://www.ivis.org>.

VALADÃO, C.A.A. et al. Analgesia epidural com xilazina: avaliação cirúrgica e hemogasimétrica. Ars Vet, v.6, n.2, p.125135, 1990.

VALADÃO, C.A.A. Avaliação da injeção peridural do amitraz em eqüinos. 1998. 63f. Tese (Livre Docência) Faculdade de Ciências Agrárias e Veterinárias, Universidade Estadual Paulista, Jaboticabal,.

VALADÃO, C.A.A. et al. Comparative study of epidural xylazine and clonidine in horses. In: WORLD CONGRESS OF VETERINARY ANESTHESIA, 8., 2003, Tenessee. Proceedings... Tenessee: University of Tenessee, 2003. V.1, p.197.

VESAL, N. et al. Epidural xylazine as an analgesic and sedative for treat surgery in cows. J Appl Anim Res, v.14, p.175-179, 1998.

WU, C.L. et al. The effect of pain on health-related quality of life in the immediate postoperative period. Anesth Analg, v.97, p.1078-1085, 2003. 